\title{
Disturbed Flow Induces Endothelial Dysfunction by Regulating Thioredoxin-Interacting Protein-Mediated Mitochondrial Energy Homoeostasis
}

\section{Yongshun Wang}

Department of Cardiology, Shenzhen People's Hospital, Shenzhen, Guangdong, China Jingjin Liu

Department of Cardiology, Shenzhen People's Hospital, Shenzhen, Guangdong, China

\section{Xin Sun}

Department of Cardiology, Shenzhen People's Hospital, Shenzhen, Guangdong, China

\section{Jie Yuan}

Department of Cardiology, Shenzhen People's Hospital, Shenzhen, Guangdong, China

\section{Huadong Liu}

Department of Cardiology, Shenzhen People's Hospital, Shenzhen, Guangdong, China

\section{Ruimian Chen}

Department of Cardiology, Shenzhen People's Hospital, Shenzhen, Guangdong, China

\section{Bihong Liao}

Department of Cardiology, Shenzhen People's Hospital, Shenzhen, Guangdong, China

\section{Haixia Gong}

Department of Cardiology, Shenzhen People's Hospital, Shenzhen, Guangdong, China

\section{Zhengyuan Xia}

Department of Anesthesiology, The University of Hong Kong, Hong Kong SAR, China

Shaohong Dong ( $\square$ forres_dsh@163.com )

Shenzhen People's Hospital

\section{Research}

Keywords: Disturbed flow, Thioredoxin-interacting Protein (TXNIP), Endothelial dysfunction, Mitochondrial dysfunction

Posted Date: January 13th, 2021

DOl: https://doi.org/10.21203/rs.3.rs-142244/v1

License: (c) (1) This work is licensed under a Creative Commons Attribution 4.0 International License.

Read Full License 


\section{Abstract}

Endothelial cells are highly sensitive to hemodynamic shear stresses, which act in the blood flow's direction on the blood vessel's luminal surface. Endothelial cells on that surface, thusly, are exposed to various physiological and pathological stimuli, such as disturbed flow-induced shear stress, which may exert effects on adaptive vascular diameter or structural wall remodeling. Here we showed that human endothelial cells exposed to disturbed flow exhibited increased levels of thioredoxin-interactive protein (TXNIP) in vitro. On the other hand, deletion of human endothelial TXNIP increased capillary formation, NO production and mitochondrial function, as well as lessened oxidative stress response and endothelial cell inflammation. Additional beneficial impacts from TXNIP deletion were also seen in a glucose utilization study, as reflected by augmented glucose uptake, lactate secretion and extracellular acidification rate. Taken together, our results suggested that TXNIP is a key component involved in mediating shear stress-induced inflammation, energy homeostasis, and glucose utilization, ultimately establishing it as a potentially novel endothelial dysfunction regulator.

\section{Introduction}

Shear stress is one of the primary mechanical forces large arteries are subjected under [1]. Mechanicallystimulated release of potent shear-responsive factors from endothelial cells regulates vessel tone and structure [2-5]. This process is facilitated by the endothelium being sensitive to hemodynamic shear stresses acting on the vessel luminal surface in the direction of blood flow. By extension, physiological and pathological variations of shear stress, caused by multiple pathophysiological conditions such as hyperlipidemia, hypertension, diabetes and inflammatory disorders, regulate endothelium-dependent changes in vascular diameter in an acute manner, and when sustained induce slowly-adaptive structural wall remodeling [6].

The varying spatiotemporal scales shear stress can be found in also contributes to regional and focal heterogeneity of endothelial gene expression. This process is important in the evolution of vascular pathology. Thioredoxin-interactive protein (TXNIP) is known to promote oxidative stress by binding and subsequently inhibiting thioredoxin activity $[7,8]$. In doing so, TXNIP is reported to influence cardiac metabolism, including mitochondrial function and glucose uptake [9]. Researchers have also demonstrated that TXNIP modulates cellular glucose utilization and mitochondrial oxidation of metabolic fuels $[5,10-12]$. On the other hand, TXNIP-null mice cannot survive prolonged fasting, exhibiting dysglycemia and dyslipidemia [11]. Besides its involvement in cellular redox and energy metabolism, increasing evidence points towards TXNIP having an important role in vascular function and inflammation process. Studies in endothelial cells showed that TXNIP promotes inflammatory response in response to disturbed flow [13] and arterial stiffness [14, 15]. This pro-inflammatory effect is confirmed by the finding that it is required for NLRP3 inflammasome activation and IL-1 $\beta$ production in cultured THP-1 cells [16]. 
However, the role of TXNIP in endothelial dysfunction is not well addressed. Given the important role of TXNIP in redox homeostasis and inflammation, we hypothesized that its ablation would protect endothelial cells from oxidative stress-induced damage and reduce vascular inflammation. In the present study, we used a disturbed-flow model to investigate the effects of TXNIP deletion on cellular redox status and inflammatory response. Our data demonstrated that TXNIP plays an important role in the development of endothelial dysfunction.

\section{Materials And Methods}

\section{Cell culture and disturbed flow treatment}

Human umbilical vein endothelial cells (HUVECs) were purchased from the American Type Culture Collection (Manassas, VA), and were grown under culturing condition with Dulbecco's modified Eagle's medium containing $10 \%$ fetal bovine serum (FBS), $50 \mathrm{U} / \mathrm{mL}$ penicillin and $50 \mu \mathrm{g} / \mathrm{mL}$ streptomycin (Invitrogen, Carlsbad, CA), as specified by the manufacturer. To initiate disturbed flow treatment, confluent HUVECs, seeded onto collagen I-coated glass slides, were assembled into flow chambers and connected to the flow system for the shear experiments. HUVECs were exposed to steady laminar flow shear stress $\left(12 \mathrm{dyn} / \mathrm{cm}^{2}\right)$, disturbed flow shear stress $\left(0.5 \pm 4 \mathrm{dyn} / \mathrm{cm}^{2}\right)$ for 24 hours.

\section{Small interfering RNA (siRNA) transfection}

SiRNAs were used to silence the TXNIP expression. The TXNIP-siRNA duplex was synthesized by Shanghai GenePharma Co., Ltd. (sense: 5'CUCCCUGCUAUAUGGAUGUTT-3'; anti-sense: 5'ACAUCCAUAUAGCAG

GGAGTT-3'). The cells, treated with either the transfection reagents (vehicle) or non-targeting siRNA (sense: 5'- UUCUCCGAACGUGUCACGUTT-3'; anti-sense: 5'-ACGUGACACGUUC GGAGGAGAATT-3'), served as controls. The cells were transfected with $200 \mathrm{nM}$ siRNA using the X-treme siRNA Transfection Reagent (Roche Applied Science, Penzberg, Germany), following the manufacturer's instructions. Three experimental groups were conducted: the treatment group of laminar flow with negative control siRNA (NF+NC-siRNA), disturbed flow with negative control siRNA (DF+NC-siRNA) and disturbed flow with TXNIP-siRNA (DF+TXNIP-siRNA)

\section{Western Blot analysis}

Protein samples with equal amount of total protein were separated on SDS-PAGE (8-15\%). The separated protein gel was then transferred to polyvinylidene difluoride membranes (Millipore, Billerica, MA, USA). After blocking with $5 \%$ non-fat milk at room temperature for 1 hour in Tris-buffered saline containing $0.1 \%$ Tween-20, primary antibody incubation (TXNIP, Cat\#: 14715; Thioredoxin 1, Cat\#: 2285; GLUT4, Cat\#: 2213; pyruvate dehydrogenase E1-alpha [PDH E1a], Cat\#: 31866; p-eNOS, Cat\#: 9571; Total-eNOS, Cat\#: 5880; NLRP3, Cat\#: 13158; VCAM-1, Cat\#: 13662; ICAM-1, Cat\#: 4915; Cleaved-IL-1ß, Cat\#: 83186 and GAPDH, Cat\#: 5174 were all from Cell Signaling Technology; Anti-Nitro tyrosine antibody [Cat\#: ab42789, 
Abcam Company]) was carried out overnight at $4^{\circ} \mathrm{C}$. Afterwards, secondary antibody incubation with a peroxidase-conjugated AffiniPure goat anti-rabbit or anti-mouse IgG was conducted for 90 min at room temperature. After washing 3 times, the membranes were subjected to ECL detection. Densitometric analysis was performed using the Tanon Gel Imaging System (Shanghai Tanon, Shanghai, China). The housekeeping gene GAPDH served as a loading control.

\section{Tube Formation Assay}

Two hundred $\mu$ of Biocoat Matrigel (Becton Dickinson) was added into each well in the 24-well plate and incubated at $37^{\circ} \mathrm{C}$ for 30 min to solidify. The same batch of Matrigel was used for all the experiments. After flow velocity treatment, cells were suspended in culture medium and plated on the Matrigel-coated plate. Gels were examined using a phase-contrast microscope equipped with a digital camera after plating. Capillary-like structures were assessed and quantified by calculating the number of junctions per field. At least 5 different viewing fields per well were analysed.

\section{Measurement of NO production}

The generation of intracellular nitric oxide (NO) was monitored using the 4-amino-5-methylamino-2,7'difluorofluorescein (DAF-FM DA) reagent (Beyotime Institute of Biotechnology). HUVECs were incubated with DAF-FM DA solution at $37^{\circ} \mathrm{C}$ for $30 \mathrm{~min}$. After washing cells three times with PBS, fluorescent intensity was determined at an excitation wavelength of $488 \mathrm{~nm}$ and an emission wavelength of $525 \mathrm{~nm}$ via a fluorescent microplate reader (SpectraMax M2, Molecular Devices Corp., USA)

\section{Mitochondrial isolation and measurement of ATP levels}

For mitochondrial isolation, HUVECs were manually homogenized using a medium-fitting glass Teflon Potter-Elvehjem homogenizer in isolation buffer (mitochondrial isolation buffer: $250 \mathrm{mM}$ sucrose, $0.5 \mathrm{mM}$ EDTA, $10 \mathrm{mM}$ Tris, and $0.1 \% \mathrm{BSA}$ at $\mathrm{pH}$ 7.4). The homogenate was then clarified through centrifuging two times at $1000 \mathrm{xg}$ for $5 \mathrm{~min}$, followed by centrifugation twice more at $11000 \mathrm{xg}$ for $10 \mathrm{~min}$. The resulting supernatant and mitochondrial pellets were collected and diluted with mitochondrial isolation buffer three times of the original volume.

Mitochondrial ATP was measured by the mitochondrial ToxGlo ${ }^{T M}$ assay according to the manufacturer's protocol. Briefly, isolated HUVECs mitochondria were plated at $1 \mathrm{mg} /$ well in both white and clear bottomed 96 -well culture plates. The assay solution $(100 \mu \mathrm{L} /$ well) was then added, and the plate was incubated at room temperature for $30 \mathrm{~min}$. Luminescence was measured using a luminometer (Molecular Devices).

\section{Mitochondrial ROS levels}

Isolated mitochondria were doubly-stained with MitoTracker Red ( $0.5 \mu \mathrm{M}$; excitation/emission 550/590 $\mathrm{nm}$,Invitrogen company) and dichlorodihydrofluorescein (DCF) diacetate (10 $\mu \mathrm{M}$; excitation/emission $488 / 535 \mathrm{~nm}$,Invitrogen company). The superoxide levels were examined according to the change in 
MitoSOX Red fluorescence using a confocal microscopy (Zeiss LSM 780). Mean values were analysed by CellQuest (ver. 5.2; DB CellQuest ${ }^{\mathrm{TM}}$ Pro)

\section{Mitochondria membrane potential}

Isolated mitochondria were stained for 30 min with $0.1 \mathrm{mM}$ tetramethylrhodamine ethyl ester (Invitrogen company excitation/emission $564 / 580 \mathrm{~nm}$ ) at room temperature and measured by flow cytometry to detect the mitochondrial membrane potential.

\section{Glucose Consumption and Lactate Secretion}

HUVECs were seeded into culture plates and incubated for 5 hours. The culture medium was then changed and cells were cultured for another 16 hours. The levels of glucose in the culture medium were measured using an assay kit from Nanjing Jiancheng Bioengineering Institute (Nanjing, China), following the manufacturer's recommendations. Lactate concentration was measured with a Lactate Assay Kit (Biovision Inc.), in accordance with the manufacturer's instructions. The glucose consumption and lactate secretion were normalized to the cell number.

\section{Electron microscopy}

To determine the mitochondrial morphology, including number, size, and shape, HUVECs were sliced and fixed in $2.5 \%$ glutaraldehyde in PBS at $4{ }^{\circ} \mathrm{C}$ overnight, then fixed under $1 \%$ osmium tetroxide in PBS for 2 hours. The sliced hearts were double-stained with uranyl acetate and lead citrate. Mitochondrial morphology was observed using an electron microscope, and the number of mitochondria was calculated using ImageJ software.

\section{Assays for glucose metabolism}

Oxygen consumption rate (OCR) and extracellular acidification rate (ECAR) of HUVECs were measured using the Seahorse XF Glycolysis Stress Test Kit on an XF24 Extracellular Flux Analyzer (Agilent Technologies), following the manufacturer's instructions. Cells were grown under standard growth conditions for 1 day prior to the metabolic analysis.

\section{Statistical analysis}

GraphPad Prism 6.0 software is used to perform statistical analyses. Data are presented as mean \pm SD. All pairs were compared to each other via either Student's t-test or least significant difference (LSD) test, as appropriate. Experimental mice groups were subject to correlation analyses through Bonferroni's post hoc test. One-way analysis of variance (ANOVA) followed by Tukey's post hoc test for multiple comparisons, were utilized for comparing multiple groups among each other. P-values $<0.05$ were considered significant.

\section{Results}




\section{Increased expression of TXNIP in disturbed flow induced endothelial dysfunction}

To assess the functional role of flow disturbances on TXNIP expression, the cultured HUVECs were exposed to disturbed flow shear stress $(0.4 \mathrm{dyn} / \mathrm{cm} 2)$ or steady laminar flow $(12 \mathrm{dyn} / \mathrm{cm} 2)$ for 24 hours. TXNIP expression levels in HUVECs were then examined by Western Blotting, which demonstrated that disturbed flow significantly enhanced TXNIP protein levels compared to laminar flow (Fig. 1A). The results also showed that the levels of Thioredoxin (TRX), referred to as a small redox protein acting as an electron donor to peroxidases and ribonucleotide reductase, had no changes under disturbed conditions compared to laminar flow. In addition, to investigate whether flow velocity affects endothelial dysfunction, we tested the ability of HUVECs to form capillary-like structures. HUVECs were seeded in Matrigel after disturbed flow or laminar flow treatment, and tube formation was examined microscopically. Further confirmation of disturbed flow inducing endothelial dysfunction through regulating TXNIP expression was queried through treating the cells with either TXNIP-siRNA or negative control siRNA (NC-siRNA). The results showed that the tube structures formed more slowly under disturbed flow conditions, whereas cells subjected to laminar flow exhibited a greater extent of capillary formation (Fig. 1B). However, TXNIP-siRNA treatment abrogated the formation of the disconnected capillary-like structures but increased the development of the proper capillary network under disturbed flow conditions (Fig. 1B). Collectively, these data suggest that disturbed flow led to a significant TXNIP expression increase, resulting in endothelial dysfunction.

\section{Disturbed flow reducedproduction of NO in a TXNIP dependent manner}

Both acute and chronic attenuation in NO production are major factors favouring endothelial dysfunction. To explore the possibility of disturbed flow regulating NO production, we measured NO and nitro-tyrosine levels in endothelial cells. The results showed that disturbed flow significantly reduced NO levels and increased nitro-tyrosine in HUVECs. By contrast, TXNIP-siRNA treatment attenuated all of these alterations in the DF group (Fig. 2A and 2B). We also measured the protein levels of endothelial nitric oxide synthase (eNOS), which is primarily responsible for vascular endothelial NO generation. Western Blot analysis showed that phosphorylation of eNOS at Ser1177 was downregulated in the disturbed flow with NC-siRNA group, compared with those in steady laminar flow group (Fig. 2C). In comparison, the protein level of eNOS at Ser1177 in the DF+TXNIP-siRNA group was upregulated compared to the DF+NCsiRNA group. These results collectively suggested that elevated TXNIP during disturbed flow contributes to eNOS depression and activity.

\section{Disturbed flow induced endothelial mitochondrial dysfunction through regulating TXNIP expression}

Endothelial dysfunction is thought to be mediated mostly by reactive oxygen species (ROS). Mitochondria are the major cellular ROS producers, due to their crucial role in energy metabolism. To explore the possibility of disturbed flow inducing mitochondrial dysfunction, we detected mitochondrial ROS in isolated mitochondria from HUVECs, with or without TXNIP expression. The results showed that disturbed flow treatment exhibited higher levels of ROS compared to laminar flow, while TXNIP-siRNA significantly reduced mitochondrial ROS levels compared to the DF+NC-siRNA group (Fig. 3A). Similarly, 
disturbed flow also reduced ATP levels compared to laminar flow (Fig. 3B). By contrast, TXNIP-siRNA treatment in the disturbed flow group resulted in significantly increased ATP levels compared to the DF+NC-siRNA group (Fig. 3B). We also observed a remarkable reduction in mitochondrial membrane potential among the disturbed flow group, compared to mitochondria isolated from laminar flow-treated cells. Conversely, TXNIP-siRNA abrogated the reduction found in the disturbed flow group compared to the DF+NC-siRNA group (Fig. 3C). Mitochondrial morphology under electron microscopy in disturbed flowtreated HUVECs showed bizarre shapes and poorly-defined cristae. Conversely, TXNIP expression inhibition led to less disorganized mitochondrial morphology compared with that of the DF+NC-siRNA group. These results therefore demonstrated that disturbed flow may aggravate mitochondrial dysfunction by distorting mitochondrial morphological features and increasing ROS levels while silencing TXNIP partially reversed the pathological response in HUVECs.

\section{Disturbed flow decreased glucose utilization by TXNIP-dependent activation}

As TXNIP has been identified as a key determinant of glucose utilization in cardiac metabolism, we investigated glucose uptake and lactate production in disturbed flow-treated HUVECs, with or without TXNIP. Glucose uptake and lactate production in disturbed flow-treated HUVECs was reduced to varying degrees compared to the laminar flow group (Fig. 3A and 3B). It is worth noting that TXNIP depletion completely abrogated the disturbed flow-induced reduction of those aforementioned metabolites compared to the DF+NC-siRNA treatment group (Fig. 4A and 4B). We further examined the impacts of TXNIP deficiency on aerobic metabolism within laminar or disturbed flow groups by measuring the oxygen consumption rate (OCR) using the XF24 extracellular flux analyze, indicating aerobic metabolism of glucose via tricarboxylic acid (TCA) cycle and mitochondrial oxidative phosphorylation. As illustrated in figure $4 \mathrm{C}$ and 4D, glucose or oligomycin (an ATP synthase inhibitor) addition triggered significant OCR increase in the laminar flow group, but only a moderate augmentation in the disturbed flow group.

Conversely, TXNIP deletion triggered significant OCR increase in the disturbed flow group compared to the disturbed flow with NC-siRNA (DF+NC-siRNA) group. In addition, to determine the effects of different flow velocities on HUVEC glycolytic flux, varying levels of flow velocity were applied to cells, with or without TXNIP-siRNA present, and the glycolytic flux was detected in DMEM assay medium following sequential addition of glucose, oligomycin and 2-deoxy-glucose (2-DG, a hexokinase inhibitor). Consistent with the OCR finding, TXNIP deletion abolished the disturbed flow-mediated extracellular acidification rate (ECAR) reduction that would otherwise be present under such conditions (Fig. 4E). The quantification demonstrated that glycolysis and glycolytic capacity were significantly decreased in the disturbed flow group, compared to the laminar-flow group. Nevertheless, TXNIP depletion under disturbed flow conditions displayed similar bioenergetic profiles of glycolysis and glycolytic capacity as under laminar flow (Fig. 4F). Thus, to better understand the specific physiological role of TXNIP in glucose metabolism, we selectively measured glucose metabolism-related gene expression in endothelial cells. We found that the glucose transporter type 4 (GLUT4), a major mediator of glucose removal from the circulation, was downregulated in disturbed flow group compared to the laminar flow group, while TXNIP deletion exhibited higher levels of this protein than the DF+NC-siRNA group (Fig. 4G). This observed GLUT4 decrease was also associated with significantly-lessened expression of PDH E1a (Fig. 4G), which 
provides the primary link between glycolysis and the TCA cycle. Together, these data indicate that elevation of TXNIP expression blunted glucose uptake, suggesting that it is the key regulator mediating glucose utilization.

\section{TXNIP activation promoted the disturbed flow-induced pro-inflammatory response}

To investigate whether TXNIP mediated HUVEC pro-inflammatory response under different flow velocities, NLRP3 and cytokine interleukin (IL)-1 $\beta$ expression were examined by Western Blotting. The expression of the cleaved form of NLRP3 and IL-1 $\beta$ was upregulated in disturbed flow-treated HUVECs but decreased upon TXNIP siRNA treatment (Fig. 5A). Application of disturbed flow, but not laminar flow, upregulated the levels of cell adhesion molecules VCAM1 and ICAM1 in HUVECs. By contrast, such increases were offset by TXNIP siRNA treatment (Fig. 5B). Taken together, these results indicated that shear stress induced HUVEC inflammation, in turn contributing to endothelial dysfunction.

\section{Discussion}

TXNIP is a key skeletal muscle regulator of glucose usage and metabolism, as well as a recently-found key inflammatory mediator [17-19] [16]. In light with the latter discovery, our findings demonstrated it serving a significant role in endothelial redox and inflammatory responses, where its ablation led to prominent reduction in cellular ROS and restoration of mitochondrial function. This reduction was found to be associated with lowered inflammatory response and NLRP3 expression. Furthermore, eliminating TXNIP also yields beneficial effects in the forms of increased glucose uptake, lactate and ECAR levels in a glucose-utilization study, as well as lowered HUVEC cell adhesion through its lowering of VCAM and ICAM expression. All these findings indicate TXNIP playing an important role in the development of HUVEC shear stress response and dysfunction. Figure 6 depicts our proposed mechanism for the dysfunctionality of endothelial cells under shear stress conditions.

Diabetic individuals, who also are more prone to atherosclerosis, demonstrate higher TXNIP expression levels. Previous studies showed that lower TXNIP expression enhanced skeletal muscle glucose uptake and improved glycaemic control [17], suggesting TXNIP inhibition being a potential diabetic intervention strategy. It was reported that endogenous NO can suppress TXNIP expression and that TXNIP facilitates nitrosative stress. However, the direct effects of TXNIP on NO regulation in disturbed flow-induced endothelial dysfunction were not investigated. In this study, TXNIP/NO interaction under shear forces was demonstrated via eNOS coupling regulation. In addition, our results indicated lower ATP levels, along with increased ROS and membrane potential depolarization in mitochondria from disturbed flow-treated cells, which were all reversed under TXNIP deletion, demonstrating the latter's potential in preventing mitochondrial dysfunction. Overall, the results from this study have further clinical and therapeutic implications, where TXNIP ablation could be beneficial in diabetics via reducing vascular inflammation and dysfunction.

TXNIP expression has been previously demonstrated to be induced by a glucose-dependent signalling pathway [20]. This connection is of significant physiological relevance, owing to the finding that after its 
induction, TXNIP negatively regulates glucose uptake $[18,21,22]$. The operation of this regulatory pathway is as follows: Cells requiring more energy or "building blocks" for macromolecular synthesis demonstrate a higher glycolytic rate, and thus a decrease of levels for certain glycolytic metabolites. Both changes are sensed by the TXNIP transcriptional machinery to repress TXNIP expression. Based on our findings, the data suggest that ectopic TXNIP expression blunts glucose uptake and lactate production. Thus, we propose that TXNIP acts as a mediator to integrate cellular metabolic activity and energy requirements with cellular glucose supply, which may have important implications for endothelial cell glucose homeostasis regulation.

Several results suggest that TXNIP is a critical target for steady laminar flow-associated antiinflammatory effects $[7,23]$. Our results show that steady laminar flow increased the ability of HUVECs to form capillary like structures, as well as lowering TXNIP expression and NLRP3-mediated inflammation.

Thus, it is likely steady laminar flow inhibits TXNIP expression, leading to the limitation of HUVEC inflammation and endothelial function improvement. This study also investigated the potential role of TXNIP in disturbed flow induced HUVEC inflammation response, where the results clearly demonstrated TXNIP ablation decreasing cellular ROS levels, as well as attenuating the stimulation of pro-inflammatory and pro-adhesion gene expression.

In summary, our data suggested TXNIP playing an important role in vascular inflammation and mitochondrial dysfunction, with respect to endothelial dysfunction development. TXNIP expression modulation could therefore be a potential therapeutic strategy for intervention in flow velocity-related vascular complications.

\section{Declarations}

\section{Ethics approval and consent to participate}

All animal care was conducted in accordance with the "Principles of Animal Care" (Ethical and Animal Welfare Committee of Heilongjiang Province, China) and were approved by the ethics review board of Jinan University and Southern University of Science and Technology.

\section{Authors' contributions}

YS W, JJ L and XS performed the experiments and were major contributors in writing the manuscript. HD $L$ and SH D did data analysis and interpretation, as well as being responsible for the study design and manuscript drafting. JY was responsible for statistical analysis. YS W prepared the reagents and revised the manuscript. $Z Y X$ and SH D designed the entire study and provided funding. All authors read and approved the final manuscript.

\section{Acknowledgements}

Not applicable. 


\section{Consent for publication}

All co-authors have read the manuscript and approved its submission to Journal of Translational Medicine.

\section{Competing interests}

The authors declare that they have no competing interests.

\section{Funding}

This work was financially supported through grants from the National Natural Science Foundation (Project Numbers 81800269, 81770496) and Shenzhen Science and Technology Plan Project (No. JCYJ20170307095947514).

\section{Data Availability Statement}

The data that support the findings of this study are available from the corresponding authors upon reasonable request.

\section{References}

1. VanEpps, J.S. and D.A. Vorp, Mechanopathobiology of atherogenesis: a review. Journal of Surgical Research, 2007. 142(1): p. 202-217.

2. Pohl, U., et al., Crucial role of endothelium in the vasodilator response to increased flow in vivo. Hypertension, 1986. 8(1): p. 37-44.

3. Moncada, S., Adventures in vascular biology: a tale of two mediators. Philosophical Transactions of the Royal Society B: Biological Sciences, 2006. 361(1469): p. 735-759.

4. Corson, M.A., et al., Phosphorylation of endothelial nitric oxide synthase in response to fluid shear stress. Circulation research, 1996. 79(5): p. 984-991.

5. Griffith, T.M., Endothelial control of vascular tone by nitric oxide and gap junctions: a haemodynamic perspective. Biorheology, 2002. 39(3, 4): p. 307-318.

6. Aird, W.C., Endothelial biomedicine. 2007: Cambridge university press.

7. Junn, E., et al., Vitamin D3 up-regulated protein 1 mediates oxidative stress via suppressing the thioredoxin function. The Journal of Immunology, 2000. 164(12): p. 6287-6295.

8. Nishiyama, A., et al., Redox regulation by thioredoxin and thioredoxin-binding proteins. IUBMB life, 2001. 52(1): p. 29-33.

9. Yoshioka, J., et al., Deletion of thioredoxin-interacting protein in mice impairs mitochondrial function but protects the myocardium from ischemia-reperfusion injury. The Journal of clinical investigation, 2012. 122(1): p. 267-279. 
10. Hui, T.Y., et al., Mice lacking thioredoxin-interacting protein provide evidence linking cellular redox state to appropriate response to nutritional signals. Journal of Biological Chemistry, 2004. 279(23): p. 24387-24393.

11. Oka, S.-i., et al., Impaired fatty acid utilization in thioredoxin binding protein-2 (TBP-2)-deficient mice: a unique animal model of Reye syndrome. The FASEB journal, 2006. 20(1): p. 121-123.

12. Sheth, S.S., et al., Thioredoxin-interacting protein deficiency disrupts the fasting-feeding metabolic transition. Journal of lipid research, 2005. 46(1): p. 123-134.

13. Wang, X.-Q., et al., Thioredoxin interacting protein promotes endothelial cell inflammation in response to disturbed flow by increasing leukocyte adhesion and repressing Kruppel-like factor 2. Circulation research, 2012. 110(4): p. 560-568.

14. Ferreira, N.E., et al., Thioredoxin interacting protein genetic variation is associated with diabetes and hypertension in the Brazilian general population. Atherosclerosis, 2012. 221(1): p. 131-136.

15. Alvim, R., et al., Thioredoxin interacting protein (TXNIP) rs7212 polymorphism is associated with arterial stiffness in the Brazilian general population. Journal of human hypertension, 2012. 26(5): p. 340 .

16. Zhou, R., et al., Thioredoxin-interacting protein links oxidative stress to inflammasome activation. Nature immunology, 2010. 11(2): p. 136.

17. Hui, S.T., et al., Txnip balances metabolic and growth signaling via PTEN disulfide reduction. Proceedings of the National Academy of Sciences, 2008. 105(10): p. 3921-3926.

18. Parikh, H., et al., TXNIP regulates peripheral glucose metabolism in humans. PLoS medicine, 2007. 4(5): p. e158.

19. Muoio, D.M., TXNIP links redox circuitry to glucose control. Cell Metabolism, 2007. 5(6): p. 412-414.

20. Yu, F.-X., et al., Thioredoxin-interacting Protein (Txnip) Gene Expression SENSING OXIDATIVE PHOSPHORYLATION STATUS AND GLYCOLYTIC RATE. Journal of Biological Chemistry, 2010. 285(33): p. 25822-25830.

21. Yoshioka, J., et al., Targeted deletion of thioredoxin-interacting protein regulates cardiac dysfunction in response to pressure overload. Circulation research, 2007. 101(12): p. 1328-1338.

22. Patwari, P., et al., Thioredoxin-independent regulation of metabolism by the a-arrestin proteins. Journal of Biological Chemistry, 2009. 284(37): p. 24996-25003.

23. Nishiyama, A., et al., Identification of thioredoxin-binding protein-2/vitamin D3 up-regulated protein 1 as a negative regulator of thioredoxin function and expression. Journal of Biological Chemistry, 1999. 274(31): p. 21645-21650.

\section{Figures}




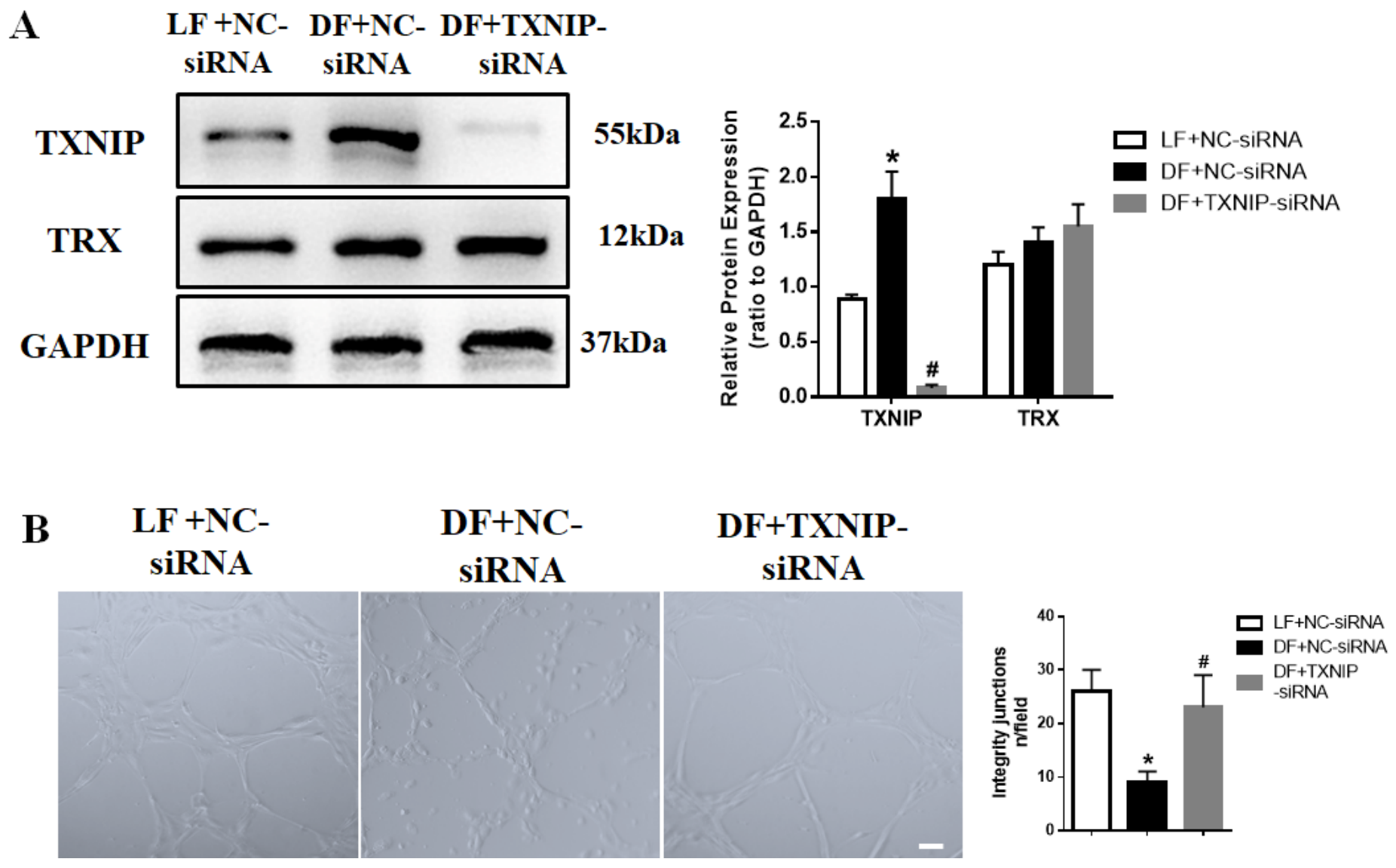

Figure 1

Increased expression of TXNIP in disturbed flow-induced endothelial dysfunction. A. Thioredoxininteractive protein (TXNIP) expression was determined by Western Blotting in the treatment groups of laminar flow with negative control siRNA (NF+NC-siRNA), disturbed flow with negative control siRNA (DF+NC-siRNA) and disturbed flow with TXNIP-siRNA (DF+TXNIP-siRNA). B. Formation of capillaries in Matrigel. Scale bars $=50 \mu \mathrm{m}$. Results are shown as mean $\pm \mathrm{SD} . \mathrm{N}=5$ /group. ${ }^{*} \mathrm{P}<0.05$ vs NF+NC-siRNA, $\# P<0.05$ vs DF+NC-siRNA. 
A

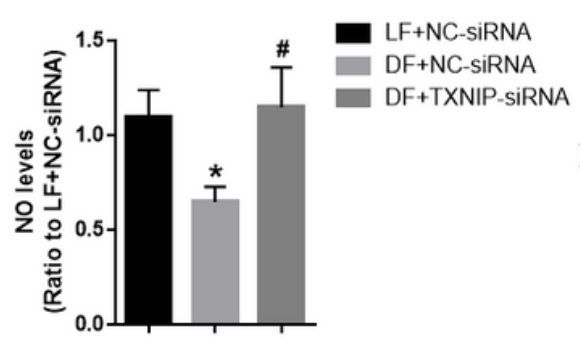

B

Nitrotyrosine

GAPDH
LF +NC- DF+NC- DF+TXNIP siRNA SiRNA -siRNA
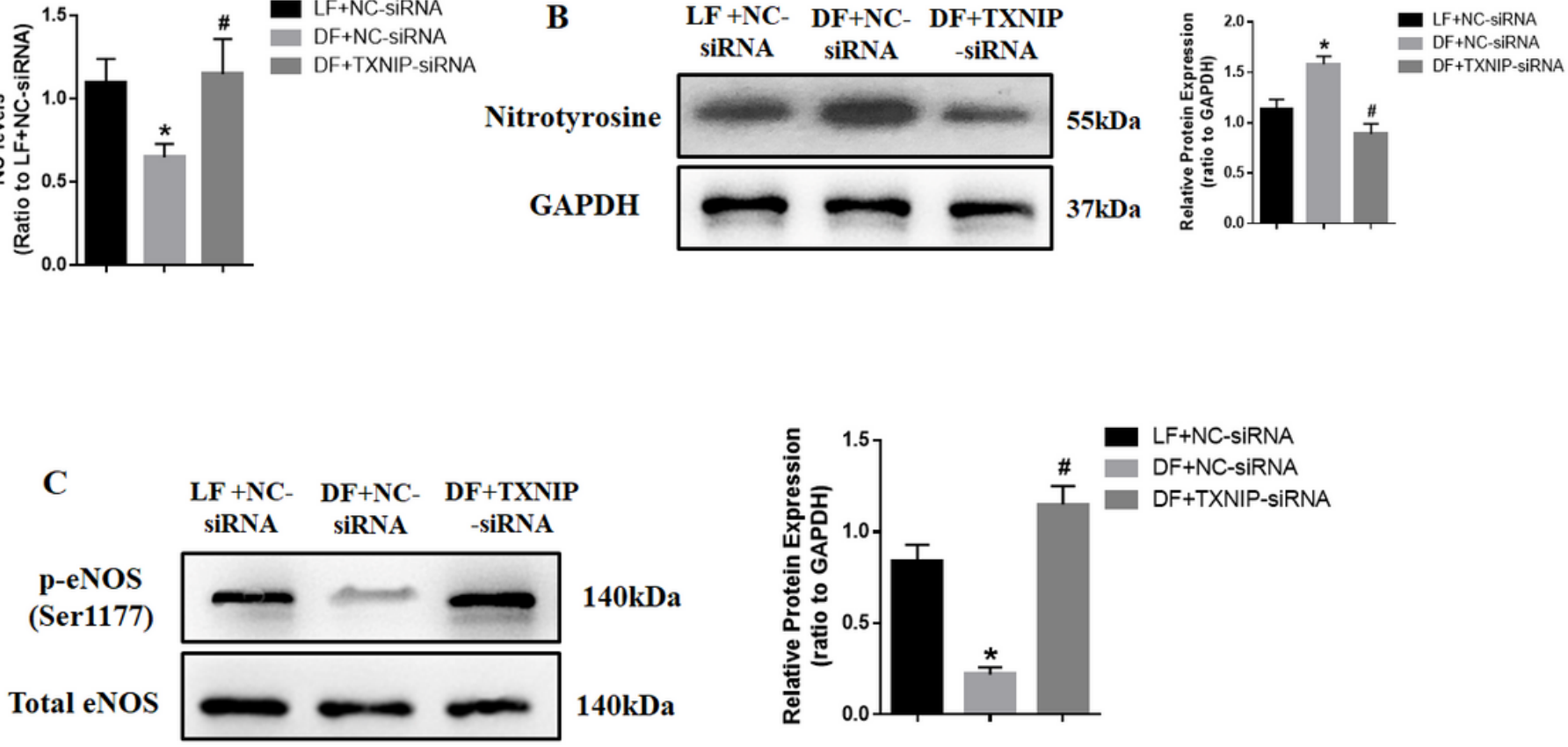

Figure 2

Disturbed flow reduced NO production in a TXNIP dependent manner A. NO production was assessed with DAF-FM DA, B. Western Blot analysis of nitro-tyrosine modified protein expression, C. Western Blots comparing the presence of phosphorylated eNOS at Ser1177 and total eNOS, in the NF+NC-siRNA, $D F+N C$-siRNA and DF+TXNIP-siRNA treated groups. Results are shown as mean $\pm S D . N=5 /$ group. *P < 0.05 vs NF+NC-siRNA, \#P < 0.05 vs DF+NC-siRNA. DAF-FM DA: 4-amino-5-methylamino-2,7'difluorofluorescein.

A

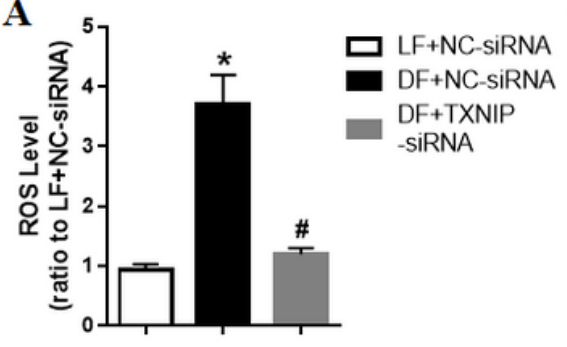

D

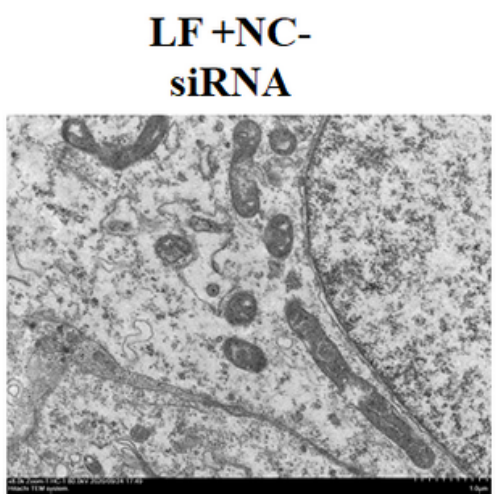

B

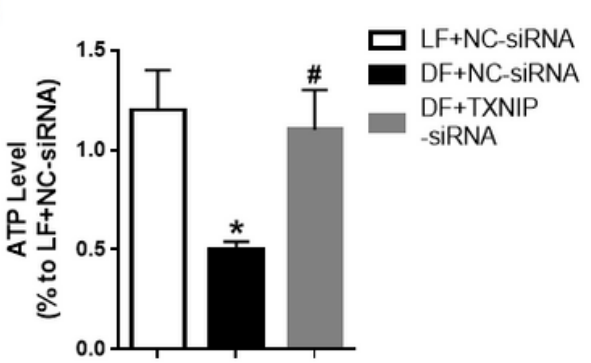

DF+NCSiRNA

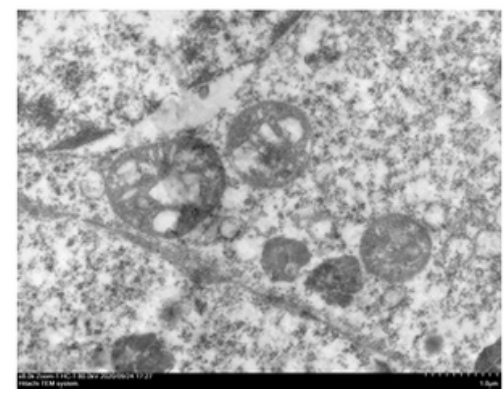

C

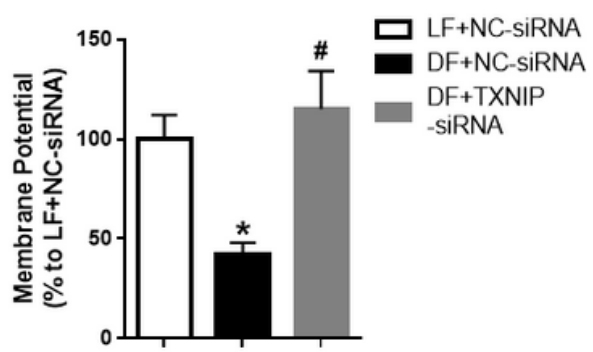

DF+TXNIP-

siRNA

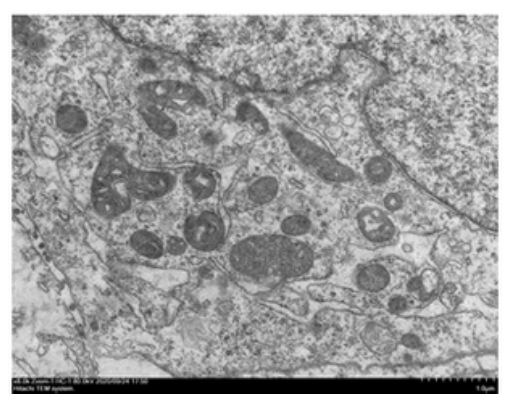


Figure 3

Disturbed flow induced endothelial mitochondrial dysfunction by regulating TXNIP expression. A.

Reactive oxygen species (ROS) level, B. Mitochondrial ATP level, C. Mitochondrial membrane potential, in the NF+NC-siRNA, DF+NC-siRNA and DF+TXNIP-siRNA treated groups. Results are shown as mean \pm SD. $N=5$ /group. ${ }^{*} P<0.05$ vs NF+NC-siRNA, \#P $<0.05$ vs $D F+N C-$ siRNA.

A

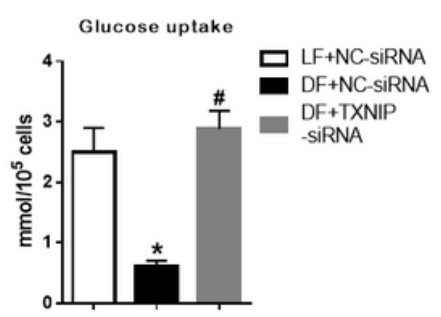

B

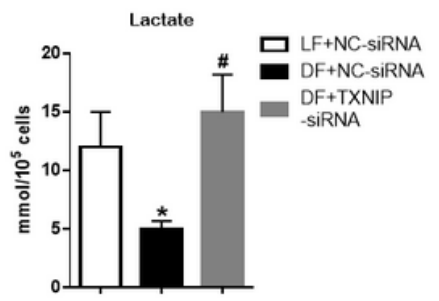

C

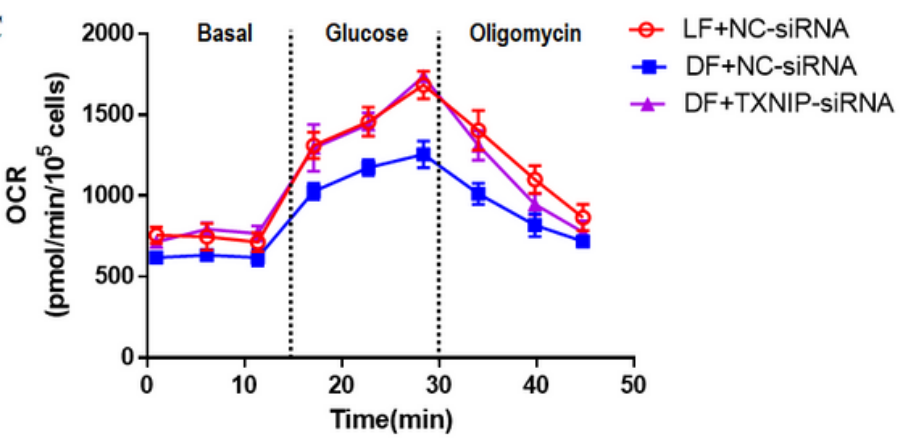

$\mathbf{E}$

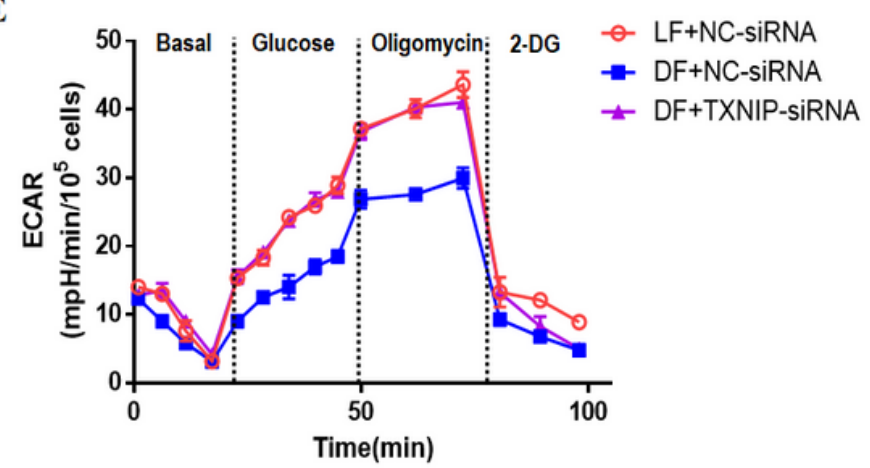

D

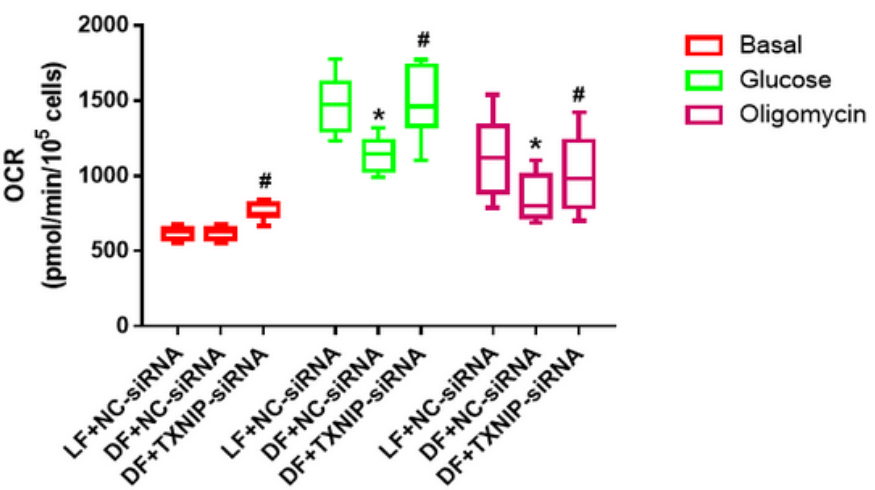

$\mathbf{F}$

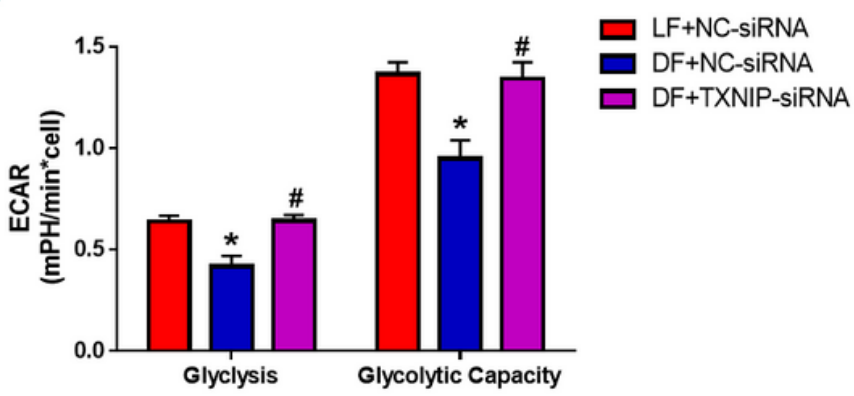

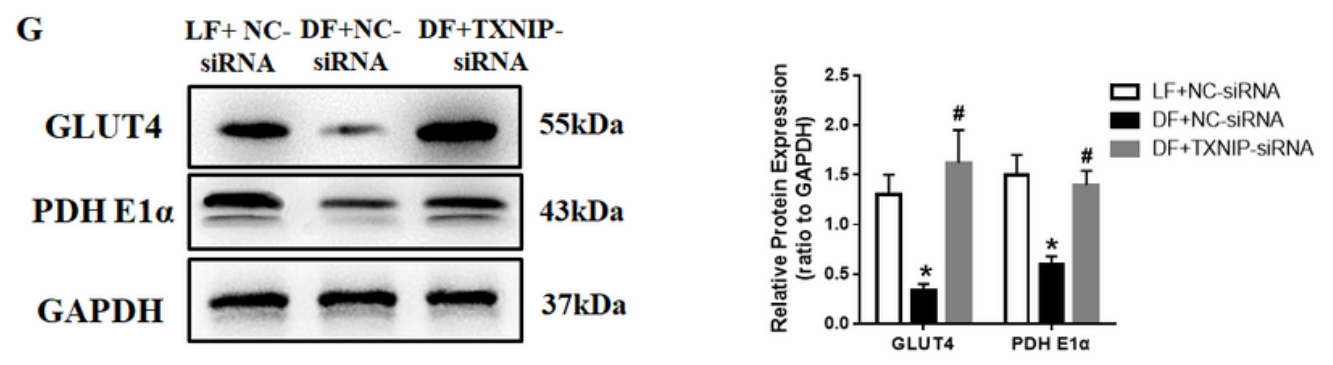

Figure 4

Disturbed flow decreased glucose utilization through TXNIP-dependent activation. A. Glucose uptake, B. Lactate production, C. Kinetic oxygen consumption rate (OCR) responses of HUVECs to $20 \mathrm{mM}$ glucose 
and $5 \mathrm{mM}$ oligomycin, D. Calculated glucose oxidation rate, E. Kinetic extracellular acidification rate (ECAR) responses of HUVECs to glucose $(20 \mathrm{mM})$, oligomycin $(5 \mu \mathrm{M})$ and 2-DG $(100 \mathrm{mM})$, in the NF+NCsiRNA, DF+NC-siRNA and DF+TXNIP-siRNA treatment groups. F. Calculated glycolytic flux and glycolytic capacity. The glycolytic flux and glycolytic capacity are calculated by ECAR increase normalized with cell protein content. All values are presented as mean $\pm \mathrm{SD}$. $\mathrm{N}=5$ /group. ${ }^{*} \mathrm{P}<0.05$ vs $\mathrm{NF}+\mathrm{NC}$-siRNA, $\# \mathrm{P}<0.05$ vs DF+NC-siRNA.

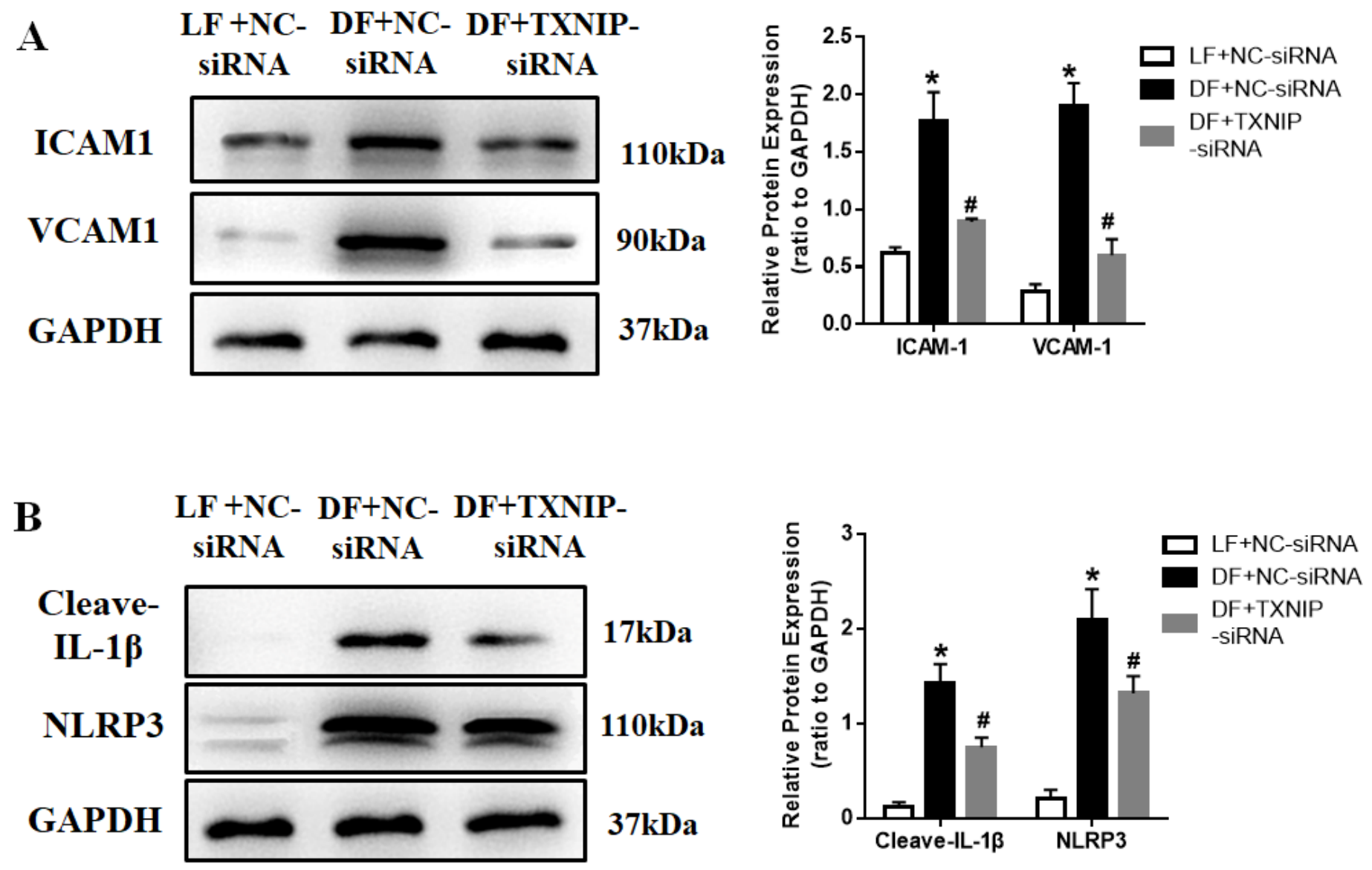

Figure 5

TXNIP activation promoted the disturbed flow-induced pro-inflammatory response. A. Representative Western Blots, along with VCAM1 and ICAM1 quantification, B. Representative Western Blots, along with quantification of cleaved-IL-1 $\beta$ and NLRP3, in the NF+NC-siRNA, DF+NC-siRNA and DF+TXNIP-siRNA groups. The blot shows representative images of five independent experiments. Results are shown as mean $\pm S D$. ${ }^{*} P<0.05$ vs NF+NC-siRNA, \#P<0.05 vs DF+NC-siRNA. 


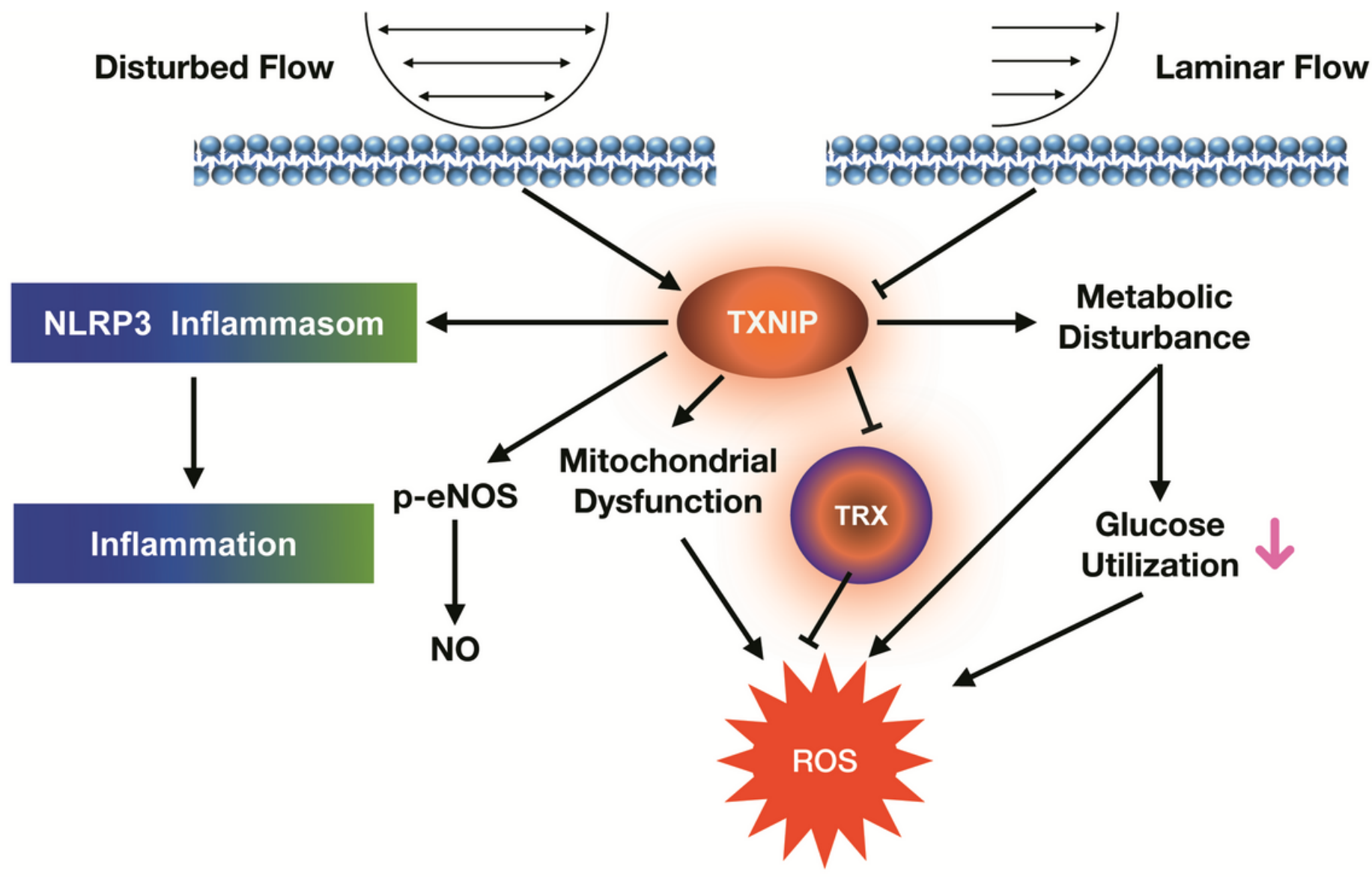

Figure 6

Proposed signalling mechanism linking shear stress to endothelial dysfunction in HUVECs 